\title{
Technique of Engineering Structures Strength Analysis Taking into Account Damageability of Materials during Creep
}

\author{
Belov A.V. \\ Kamishin technological institute (branch), Volgograd state \\ technical university \\ Kamishin, Russia \\ end@kti.ru
}

\author{
Morozova E.V., 1,2 \\ ${ }^{1}$ Kamishin technological institute (branch), Volgograd state \\ technical university \\ Kamishin, Russia \\ ${ }^{2}$ Saint Petersburg Electrotechnical University "LETI" \\ Saint Petersburg, Russia \\ end@kti.ru
}

Polivanov A.A.

Kamishin technological institute (branch), Volgograd state technical university

Kamishin, Russia

end@kti.ru

\begin{abstract}
This work describes research methods of complex problem solution to estimate strength and durability of flat laminas and multiple layer shells of rotation with an account of plastic deformations, temperature creep, the hydrogen corrosion and degradation of material's properties in time. The practical solutions of the problems of calculating the stress-strain state of a flat lamina with a hole was made. The lamina was under pressure at a temperature of $500^{\circ} \mathrm{C}$. The dependence of the time of the onset of the limiting state on the value of the damage parameter for three different pressure values is investigated. The components of the stress-strain state and the time to failure of the laminas were determined. As the limiting state, the moments of the onset of the process of destruction and complete destruction of the laminas were considered. In the course of the research it was found that the choice of the damage parameter limiting value has a significant effect on the fracture time evaluation, and this effect is especially pronounced when performing calculations taking into account the motion of the fracture front. If the damage parameter is incorrectly selected, the time to failure estimate can be either overestimated or underestimated. On the basis of the obtained results of calculations, recommendations are formulated for the choice of the adequate damage parameter values.
\end{abstract}

Keywords-steel shells; high temperature corrosion; high temperature creep; elastoplastic deformation

\section{INTRODUCTION}

The most important problem of energy and petrochemical as well as aircraft engineering development is the creation of new generation arrangement rendering possible realization of strict operating practice of exploitation (high temperature, pressure, speed, environmental aggressiveness, etc.) and providing high reliability and specified life. For designing such constructions the
Neumoina N.G.

Kamishin technological institute (branch), Volgograd state technical university

Kamishin, Russia

end@kti.ru

elaboration of design complexes rendering possible to carry out the investigation and behavior simulation of such constructions taking into account the attack of non-steady temperature, force field and degradation of mechanical-and-physical properties of their materials. The specified complexes including quite a number of basic subsystem's should operate from mathematical models describing the evolution of stress and strain state of the constructions under the conditions of their near-real exploitation

In this connection the elaboration of system concept of construction and further using of such design complexes both from methodological point of view and from the position of construction of integrated functional design of interdependent models rendering possible to solve complex problems passing from their simple statement to a more complicated one becomes of the current interest [1]. At that the models determining the structures behavior and integrated into a complex are constructed with due consideration of present-day state of theoretical research and experimental data on fullness and adequacy of the considered physical and mechanical processes description [3, 4]. If necessary the models are complicated on account of their correlation. For this a hierarchic approach to the formation of model system realizing the principle "from simple to complex" is used, when the next difficulty level is reached after detailed enough study of a more simple model. Hence, a multilevel architecture of more and more complex models every one of which correlates the previous ones including them as special cases.

\section{THE PROBLEM'S STATEMENT}

Thus, the most productive approach to solving these problems in terms of efficiency and reliability of the results is loading $[1,2]$. 
the approach of the development of methods for studying and modeling thermo-viscoelastoplastic stress-strain state of inhomogeneous multilayer shell structures during accounting materials damage of under creep [5].

The authors propose a methodology, including the joint use of software complex, based on the solution of a certain class of tasks (shell of rotation), as well as other packages, in particular ANSYS, as much as possible fully using the strengths of all program complexes [6]. In this case, the application of the developed software package allows adding other factors of influence in the modeling process.

\section{THE DESCRIPTION OF CALCULATION'S METHODS}

As a result of a phased application of the system approach, a multi-level architecture of models is built, each of which generalizes the previous ones. Currently, the developed calculation method makes it possible to solve problems in the statement of the problem:

\section{Thermo-elastic statement of the problem.}

\section{Thermo-elastic-plastic statement of the problem.}

3, Thermo-viscoelastoplastic statement of the problem ignoring materials damageability at creeping.

4. Thermo-viscoelastoplastic statement of the problem inclusive materials damageability at creeping.

5. Thermo-viscoelastoplastic statement of the problem inclusive materials damageability at creeping with fracture propagation stage investigation.

When solving the problem in thermo viscoelastoplastic formulation, taking into account the material damageability under creep, the relations of the theory of non-isothermal processes of solid elements elastoplastic deformation along trajectories of small curvature linearized by the method of additional deformations are used as determining equations. To take into account the damage that occurs in the materials of the structure during the creep process, effective stresses are used, that is, stresses calculated taking into account the resulting damage to the material. These damages are taken into account with the help of the scalar parameter of damage. To determine the damage parameter, the Rabotnov's kinetic equation of damping is used [8]:

$$
\frac{d \omega_{C}}{d t}=C_{i}\left(\frac{\sigma_{\ni}}{1-\omega_{C}}\right)^{Q_{i}}
$$

Here $\omega_{C}$ is the damaging parameter determining the degree of material damage in the shell element; $\sigma_{\ni}$ is the equivalent voltage, by means of which the influence of the stress state of the shell element on the development of the accumulation of damage is taken into account; $C_{i}$ and $Q_{i}$ are coefficients that are some characteristics of the material, temperature dependent, which are determined on the basis of the long-term strength diagrams, experimentally obtained for a number of fixed temperatures.
As the equivalent stress one of the criteria of long-term strength - Johnson, Kats, Sdobyrev, Trunin or LebedevPisarenko - can be used [7]. The choice of a creep rupture strength criterion describing the material's damageability process at creeping most adequately depends on the kind of stress state, stress level and availability of enough test data.

The process of damage accumulation in the material of the studied shell is calculated by means of successive solutions of the kinetic damageability equation in every loading step. The investigation of the damage accumulation process in the shell element lasts until achieving a preset limit value close to the unit by the $\omega_{c}$ parameter. It is the condition of the shell's local fracture e, i.e. fracture process beginning.

For an approximate evaluation of the time to complete destruction, the L.M. Kachanov's method to analyze of the development fracture region kinetics is used [9]. It is based on the study of the displacement process of the destruction front (the surface is separating the undestroyed and destroyed areas of the material). The kinetics of the development scheme analysis of the fracture region is as follows. Until the beginning of the destruction process, it is assumed that there are no destroyed elements in the construction. At the moment of the local destruction, the most loaded individual elements of the construction are rapidly destroyed and, thus, in addition to the zone of undamaged material, a destruction zone is formed. As a result, the entire load is redistributed among the elements that are part of the undamaged area. With further loading of the construction, the destruction zone increases in size, which will manifest itself in the motion of the destruction front. If the size of the destruction area increases so much that the rest of the undamaged material can no longer withstand the external load, a complete and almost instantaneous destruction of the construction occurs. This time was called the time of total destruction.

A detailed description of the methods for solving each of the above problems is given in [2].

This approach was realized by the authors with reference to thin single-layered and multi-layered circular flat laminas and shells of rotation [7].

In addition, if the constructions under consideration are under the influence of a hydrogen-containing medium, then all the above-mentioned problems can be solved taking into account high-temperature hydrogen corrosion. For this, the calculation procedure is supplemented by a generalized model of the effect on the construction of a hydrogen-containing medium [10].

In this model it is assumed that the effect of hydrogen on the mechanical properties of low-carbon steels is manifested after a several time, called the incubation period $t_{i n c}$. After its completion, intensive decarburization of the construction material is begins, accompanied by a degradation in its mechanical properties. In this case, the mechanical properties of materials can vary significantly. This period is called the period of active chemical transformations $t_{c r}$. Numerous researchers have established that, for $t<t_{i n c}$, there is no change in the mechanical properties of materials yet, and at $t>t_{c r}$ no longer occurs [10]. The process of active chemical 
transformations takes a several time, and this time depends on the hydrogen pressure, temperature, stress state and material damage. The change in the mechanical properties of lowcarbon steels in the decarburization process begins and ends under certain conditions that do not depend on the hydrogen pressure and temperature. The increase in pressure and temperature only leads to the fact that these conditions occur earlier, and the final state of the mechanical properties of the material is practically the same in all cases [10]. Given these assumptions, the calculation of the hydrogen corrosive effect is greatly simplified.

Thus, the influence of hydrogen on the material properties will be described in the form of a piecewise linear law for the hydrogen chemical interaction parameter $\mu$ varying from 0 to 1 and characterizing the degree of damage to the material due to hydrogen corrosion:

$$
\mu t>=\left\{\begin{array}{cc}
0, & \text { if } t<t_{i n c} \\
t-t_{i n c} / t_{c r}-t_{i n c}, & \text { if } t_{c r}<t<t_{i n c} \\
1, & \text { if } t \geq t_{c r}
\end{array}\right.
$$

The incubation period duration, as shown in [10], is determined by the following equation:

$$
t_{i n c}=\mathrm{m} \cdot \mathrm{p}^{-\mathrm{u}} e^{\frac{B}{T}}
$$

where: $p$ is the hydrogen partial pressure, $\mathrm{MPa} ; T$ is the temperature; $m, u, B$ are some constants, experimentally determined for different steel grades.

The duration of the active chemical transformations period is determined as follows [10]:

$$
t_{c \mathrm{r}}=\lambda \cdot t_{i n c}
$$

where: $\lambda$ is a parameter that is a measure of the hydrogen chemical interaction rate with the construction, showing how many times the time to complete the period of changes in the material properties differs from the duration of the incubation period.

\section{THE RESULTS OF EXPERIMENTAL STUDIES}

As an example, let us consider the stress-strain state of a two- and three-layer flat annular lamina with a round hole, which is uniformly heated to $500^{\circ} \mathrm{C}$ and under a hydrogen pressure. The geometrical dimensions of the lamina are as follows: $R=110 \mathrm{~mm}, r=65 \mathrm{~mm}, \delta=10 \mathrm{~mm}$ (Fig. 1). In a three-layer lamina, the middle layer is made of steel 20, and the upper and lower layer is made of the $12 \mathrm{~h} 18 \mathrm{n} 9 \mathrm{t}$ alloy, in a two-layered lamina, the $12 \mathrm{~h} 18 \mathrm{n} 9 \mathrm{t}$ alloy is placed on top. The hydrogen pressure is carried out from the side of the upper layer of the plate and is permanently in the time, and the inner and outer annular circuits have a hinged-fixed fixation. The constants determining the properties of the material in relations Eq. (3) and Eq. (4) were taken from [10], which for steel 20 and the loading conditions under consideration are: $\mathrm{u}$ $=1.73 ; \mathrm{B}=13500 ; \lambda=5$. Using the results of [10], it is possible to assume that the mechanical characteristics of the $12 \mathrm{~h} 18 \mathrm{n} 9 \mathrm{t}$ alloy under the influence of hydrogen remain practically unchanged during the whole considered loading period. The effect of the stress state type on the rate of the decarburizations process in multilayer plates considered in this publication is not yet possible, since additional research is required for this.

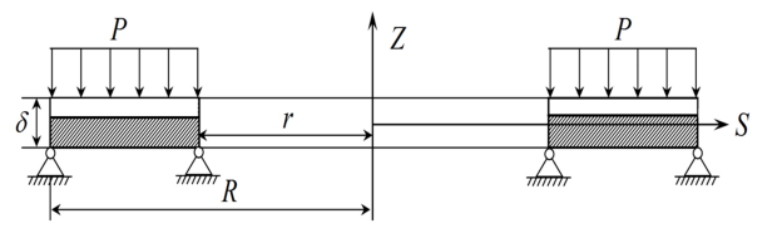

Fig. 1. The axial section of the lamina

In previous works, the authors investigated the stress-strain state of a single-and double-layer lamina, taking into account the temperature creep and hydrogen corrosion at one fixed value of the damageability parameter. In this work, we perform a similar study of a three-layer lamina of the same size and compare it with the results for a two-layer lamina. During the work, the influence of the $12 \mathrm{~h} 18 \mathrm{n} 9 \mathrm{t}$ alloy protective layer of fixed thickness on the long-term strength of the lamina was studied depending on the hydrogen pressure, as well as the distribution of deformations, stresses, damaging and plasticity zones at the time instants at the beginning of the loading process, before destruction and immediately after it at a fixed value of the damaging parameter is 0.99 .

In addition, the dependence of the calculated values of the long-term strength of the lamina on the specified limit values of the damaging parameter, as well as the dynamics of the damage parameter at the most stressed points, both before the onset of the destruction process, and with the study of the stage of failure destruction, was investigated. Some of the results obtained are shown in Fig. 2 - 7.

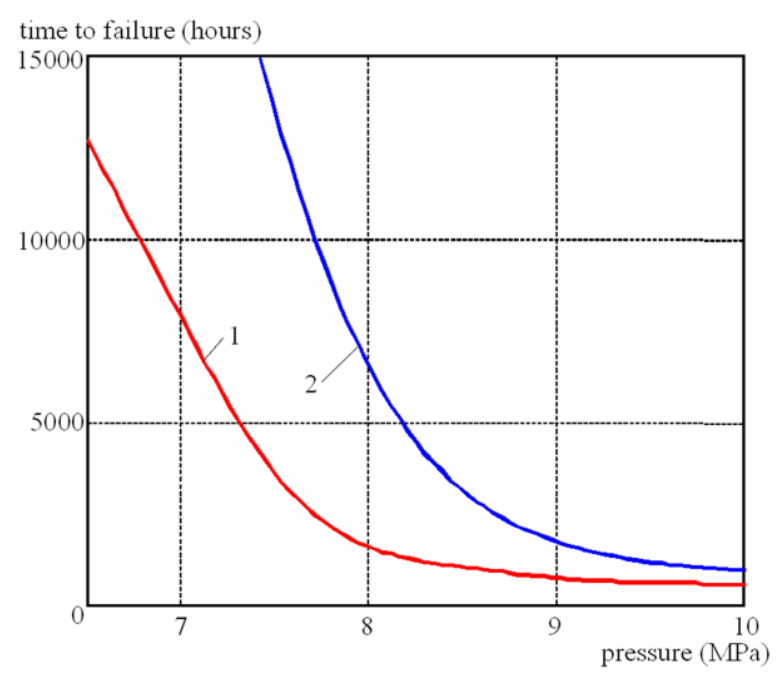

Fig. 2. The time dependence to failure on hydrogen pressure 
Fig. 2 shows the curves reflecting the time dependence to failure on hydrogen pressure, curve 1 - a two-layer plate, curve 2 -three-layer. Fig. 3 shows the cuts of the lamina axial section, on which the distribution of damage zones by the gradation of the gray color is presented at the time of the fracture process onset. The left part of the figure shows the results for a two-layer lamina, on the right side - the results obtained for a three-layer lamina.

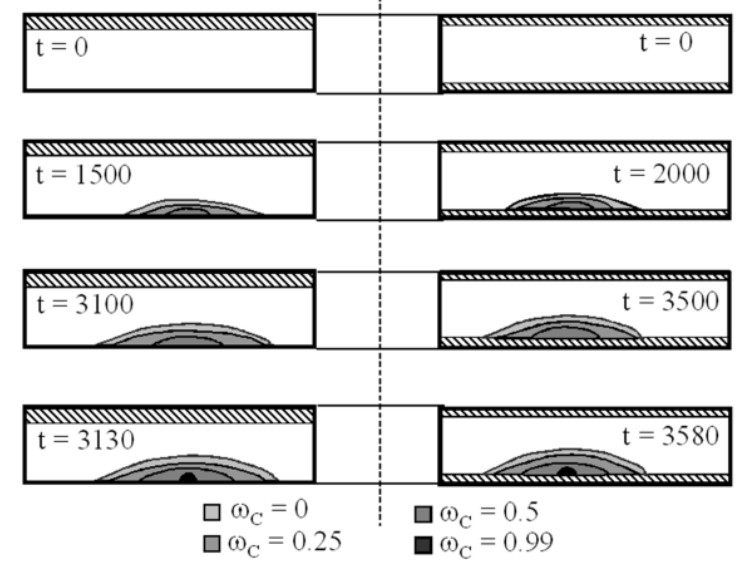

Fig. 3. The axial section of lamina with the distribution of damage zones

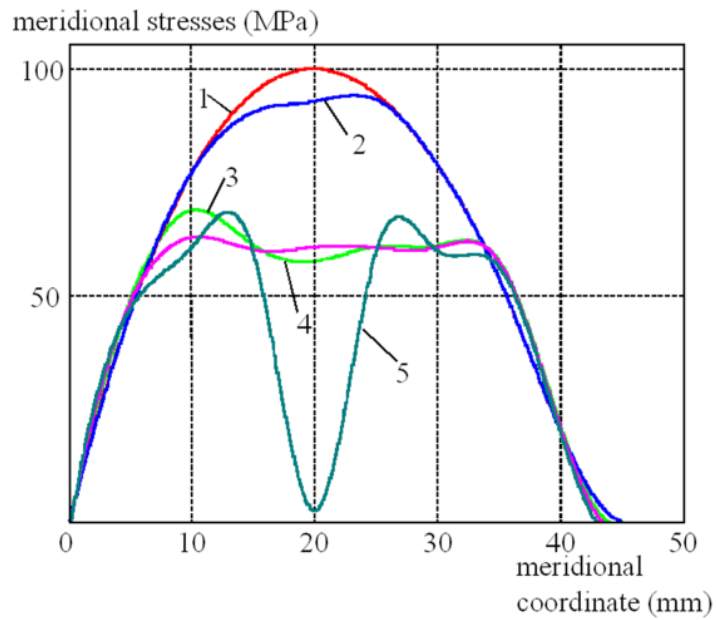

Fig. 4. The meridional stresses acting on the lower surface of the two-layer lamina

Fig. 4 shows the meridional stresses and in Fig. 5 there are circumferential stresses acting on the lower surface of the lamina, which are obtained for the same moments of time as in Fig. 6, and in Fig. 7 - similar graphs for a three-layer lamina.

Analyzing the above results, we can conclude that the nature of the damage zones distribution with the addition of the third layer looks almost identical, given that the time for achieving the same state in the three-layer lamina is greater, and the areas of material damage are always located in the layer with low-carbon steel.

Then, let us consider the results of the study of the limiting state onset time dependence (that is, local or complete destruction of the construction) from the chosen value of the damaging parameter.

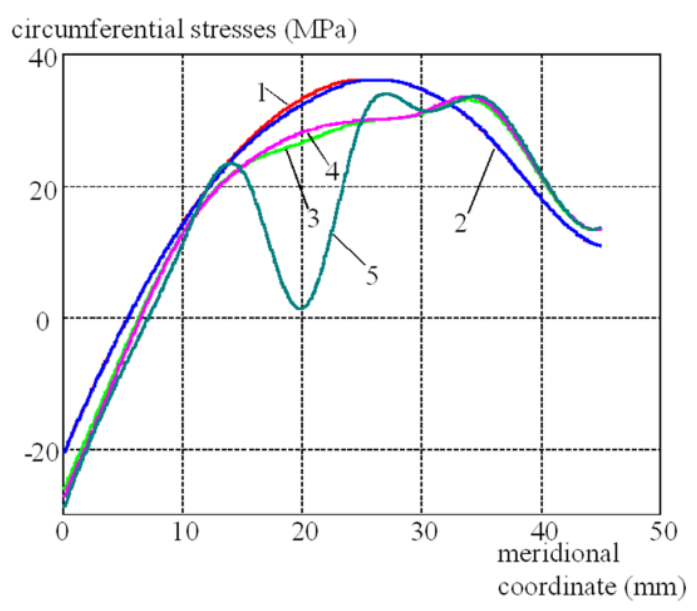

Fig. 5. The circumferential stresses acting on the lower surface of the twolayer lamina

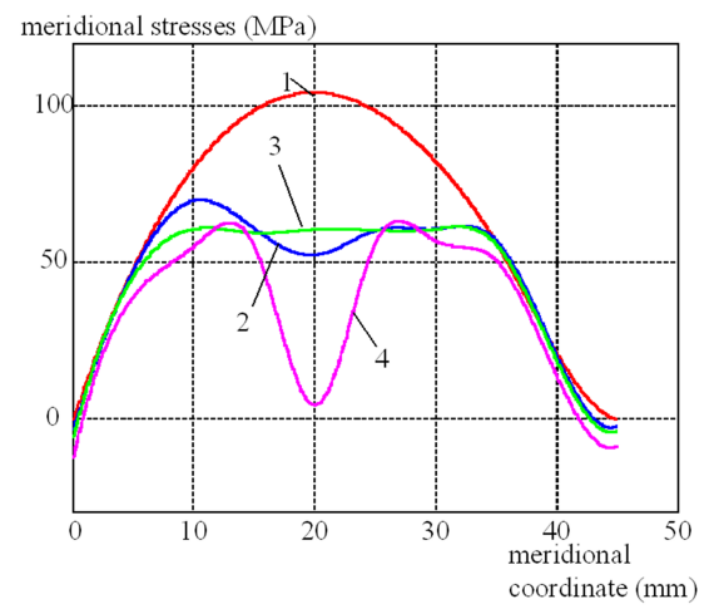

Fig. 6. The meridional stresses acting on the lower surface of the three-layer lamina

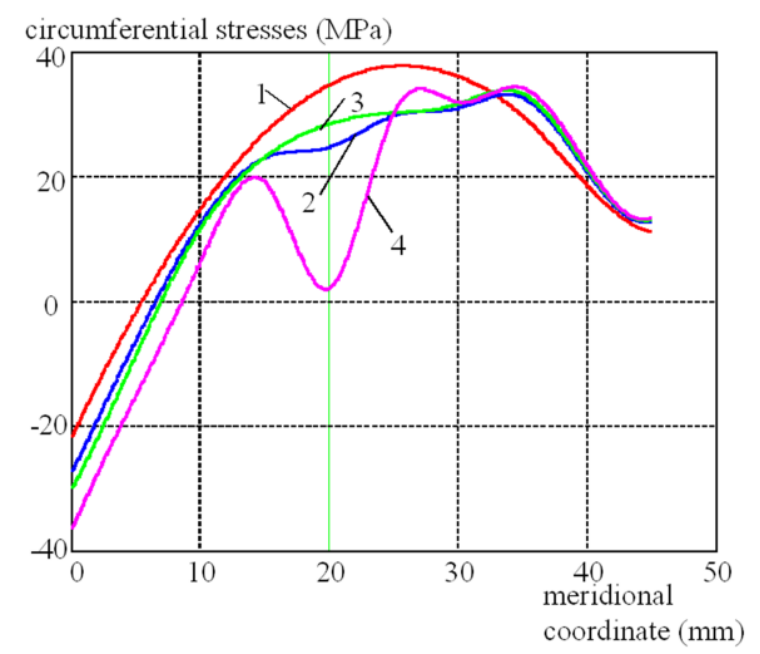

Fig. 7. The circumferential stresses acting on the lower surface of the threelayer lamina 
Fig. 8 shows the curves of the time dependence before the beginning of the damaging process of lamina from the specified value of the damaging parameter for three different pressures; curve $1-\mathrm{P}=9.5 \mathrm{MPa}$, curve $2-\mathrm{P}=8.5 \mathrm{MPa}$, curve $3-\mathrm{P}=7.5 \mathrm{MPa}$.

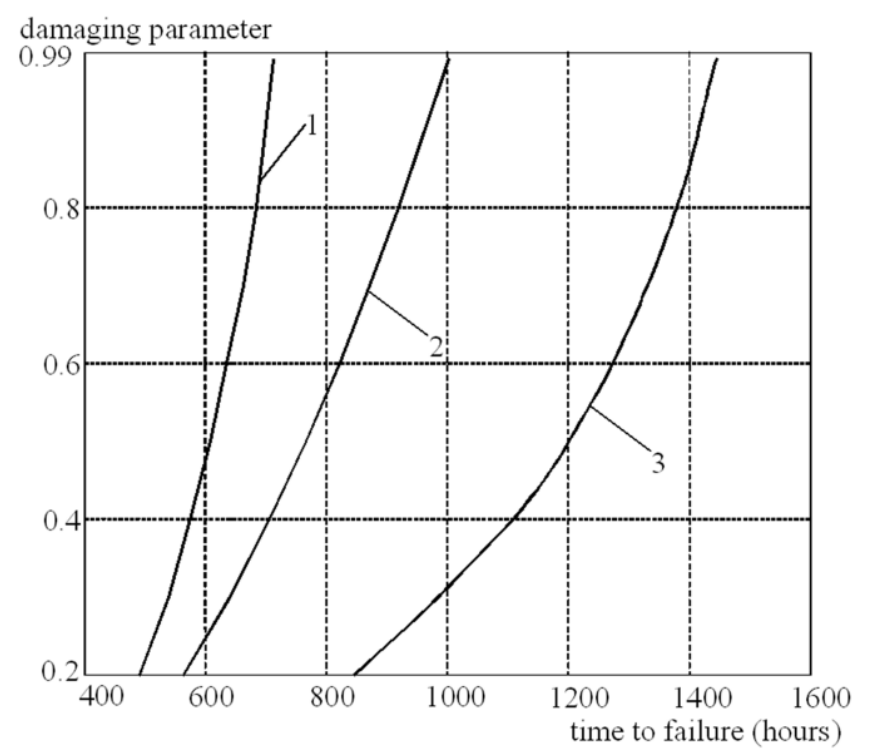

Fig. 8. The time dependence before the beginning of the damaging process of lamina from the specified value of the damaging parameter

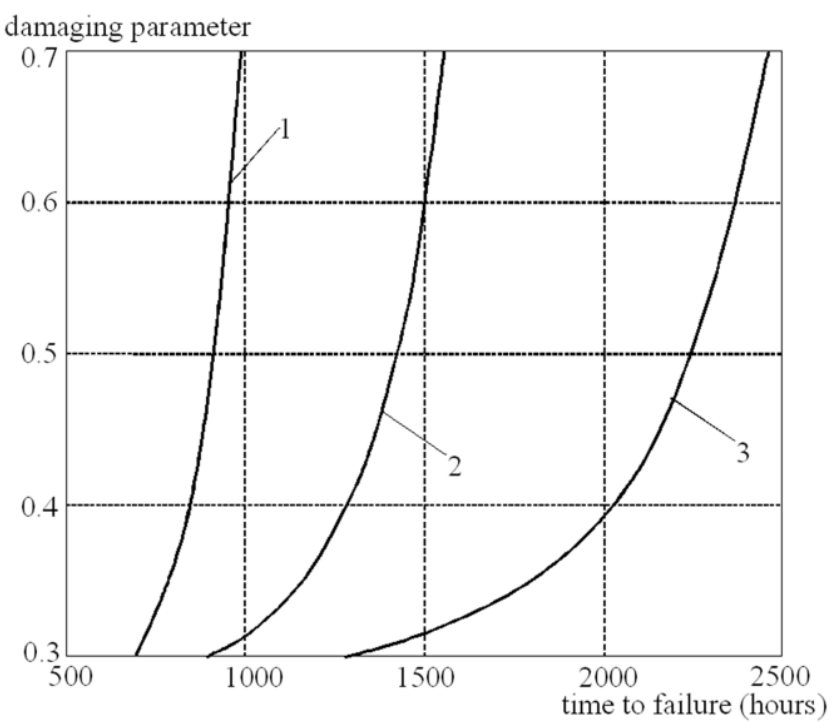

Fig. 9. The time dependence before the lamina is completely destroyed from the specified limiting value of the damaging parameter

Fig. 9 shows the curves showing the time dependence before the lamina is completely destroyed from the specified limiting value of the damaging parameter, for the same pressure values as in Fig. 6.

Based on the analysis of the results shown in Fig. $6-7$, it can be concluded that the choice of the limiting value of the damaging parameter affects the fracture time evaluation of the lamina, and this effect is especially pronounced when performing calculations taking into account of the fracture front motion. This dependence has a pronounced nonlinear character, and when a certain state is determined by a combination of several factors (stress-strain state, distribution of the temperature field, force loads, the presence of an aggressive medium, etc.), the value of the damaging parameter at certain, most loaded points begins rapidly increase and reaches its limiting value relatively quickly. It is obvious that additional experimental studies are required for a reliable choice of the damaging parameter's limiting value, for each material under consideration and loading conditions. And in the absence of these results, it is advisable to carry out a calculation of the construction's stress-strain state and time prior to the onset of failure, at least at two limiting values of the damage parameter, minimum and maximum. It is obvious that when calculating the structures for long-term strength, taking into account the motion of the fracture front, the maximum value of the damaging parameter should not be chosen close to unity.

\section{CONCLUSIONS}

The results obtained in this work show that the productive way of calculating the long-term strength and estimating the remaining resource of a wide class of engineering constructions is the use of software products, using different models of material and geometry, and the subsequent joint analysis of the calculation results. Under certain conditions, which are impossible to predict in advance, the shells calculation for strength and durability without the influence of factors, such as damage at the creep and at high-temperature hydrogen corrosion may be at incorrect results. The technique for solving the problems of strength, stiffness and durability of structural elements in the form of flat plates and thin shells of revolution with a complex form of a meridian, presented in this article, makes it possible to determine the elastoplastic stress-strain state of such shells, taking into account the material damage under creep, the kinetics of the fracture regions development and time of local and complete destruction, including the specified limit value of the damaging parameter.

\section{References}

[1] A.Z. Galishin, "Determination of the thermocomposoplastic state of the shells of revolution, taking into account the material damageability during creep" J Applied mechanics, Vol. 40, 2004, No. 5, pp. 71-79.

[2] A.V. Belov, A.A. Polivanov, E.V. Morozova, "Evaluation of StressStrain State of Engineering Structures Subject to Damage in Materials under Creep-Based Simulation" J Procedia Engineering. Vol. 206 : International Conference on Industrial Engineering (ICIE 2017) [SaintPetersburg, Russian Federation, May 16-19, 201], 2017. pp. 1464-1469..

[3] P.S. Volegov, D.S. Gribov, P.V. Trusov, "Damage and destruction: classical continuum theories", J Physical mesomechanics, Vol. 18, 2015, № 4, pp. 68-87.

[4] P.S. Volegov, D.S. Gribov, P.V. Trusov, "Damage and destruction: review of experimental works", J Physical mesomechanics, Vol. 18, 2015, No 3, pp. 11-24.

[5] R.A. Arutyunyan, "Embrittlement and destruction of metals under hightemperature creep conditions", Bulletin of the Kyrgyz-Russian Slavic University, 2017, Vol. 17, No 1, pp. 8-12.

[6] A.L Volynin, "Comparative calculation of strength and stability of reinforced shells with PC OBOLOCHKA and PC ANSYS", Bulletin of Civil Engineers. 2010, Vol 2 (23), pp. $38-43$. 
[7] V. Bagmutov, A. Belov, A. Polivanov, "Damage Calculation Features of Multilayered Shells of Rotation at Thermo-viscoelastoplastic Strain", J Mechanika, 2004, No 3(47), pp. 19 - 23.

[8] Yu.N. Rabotnov, The creep of structural elements. M.: Science. 1966, 752 p.
[9] L.M. Kachanov, Fundamentals of fracture mechanics. M.: Science. 1974, $311 \mathrm{p}$.

[10] I.G. Ovchinnikov, T.A. Hvalko, The functionality of structures in a high-temperature hydrogen corrosion. Saratov. 2003, 176 p. 